9th A. Friedmann International Seminar and

3rd Casimir Symposium 2015

International Journal of Modern Physics: Conference Series

Vol. 41 (2016) 1660146 (8 pages)

(C) The Author(s)

DOI: $10.1142 /$ S2010194516601460

\title{
Nuclear-resonance magnetometer with flowing liquid for superstrong inhomogeneous fields measuring
}

\author{
V. V. Davydov*, V. I. Dudkin and V. A. Vologdin \\ Institute of Physics, Nanotechnology and Telecommunications, \\ Peter the Great Saint Petersburg Polytechnic University, \\ 29 Politekhnicheskaya street, Saint Petersburg, 195251, Russian Federation \\ *davydov_vadim66@mail.ru
}

Received 19 September 2015

Revised 27 September 2015

Published 18 March 2016

\begin{abstract}
Multichannel nuclear-resonance magnetometer for remote monitoring of induction and heterogeneity of a magnetic field in different areas inside and near a charged particle accelerator is considered. The maximal distance between the nuclear magnetic resonance signal detector and the magnetometer is $50 \mathrm{~m}$. Measurement error is $0.5 \%$, sensitivity of the magnetometer is $10^{-10} \mathrm{~T} / \mathrm{Hz}^{1 / 2}$, measurement time of the magnetic field parameters in 24 control points is no more than 4 minutes.
\end{abstract}

Keywords: Nuclear magnetic resonance; nuclear-resonance magnetometer; accelerator; flowing liquid; magnetic field.

PACS numbers: 07.55.Ge, 06.20.Dk, 07.05.Dz

\section{Introduction}

Precision magnetic fields measurements are used in fundamental researches as well as in engineering ones ${ }^{1-4}$ and form a rapidly developing metrology branch. A part connected with strong and superstrong magnetic fields measurement holds a specific place. In the recent years the appearance of superconduction magnets attracted interest to the use of strong and superstrong magnetic fields in different scientific researches, this resulted into high accuracy requirements for measurement of these fields physical quantities (induction and discontinuity). ${ }^{3-5}$

At the present time to measure strong and superstrong magnetic fields quantities, gaussmeters (for the fields with $\mathrm{H}$ under $60 \mathrm{kG}$ ), fitted with Hall sensor, and vibration magnetometers (e.g. Lakeshore) are mainly used. Optical meters (sensors)

This is an Open Access article published by World Scientific Publishing Company. It is distributed under the terms of the Creative Commons Attribution 4.0 (CC-BY) License. Further distribution of this work is permitted, provided the original work is properly cited. 
which operating principle, based on the Faraday effect, has differed slightly since their development, are now used more rarely. ${ }^{3-7}$ At the present time magnetometers with the use of modern ferromagnetic materials for measurement of superstrong magnetic fields induction are developed at the Ioffe Physical Technical Institute.

But there are some problems to solve which the permanent control of magnetic field parameters — induction and discontinuity — in various points (from 20 to 50) almost equally (at least once in $2-4 \mathrm{~min}$ ) is required. One of the most important of these problems is control of magnetic system work, intended to form and analyze charged-particles beams. Depending on the facility modification, this system consists of 8-32 focusing magnetic quadrants, forming a cut storage circle, and 4-12 sectoral continuous injection chain electric magnets, positioned in the charged particles accelerant bunker. Under the conditions of accelerator work all the magnetic facility is based in the radiation zone, where the human appearance is impossible. According to this the accelerator magnetic system field parameters control has to be remote. Moreover, the electronic part of measurement equipment also needs to be removed from the coverage area of strong dissipated magnetic, electric and electromagnetic fields of the accelerator behind the protective structures, in other words into the accelerator control unit, where the servicers are based. In this case, the distance between the magnetic field safety points and the electronic part of measurement equipment may be from 30 to $50 \mathrm{~m}$.

The use of gaussmeters with Hall sensors with such length of measurement probe becomes an egg dance problem under conditions of different disturbances. In this case from 20 to 50 gaussmeters, depending on measuring points number, have to be involved to measure the field. Moreover, thermal discontinuity, caused by heating of some construction elements of the accelerator, may make additional problems during the Hall sensor calibrating factor retraining, solution of which may require the additional unit for every gaussmeter, used in the measurement. Optical meters (sensors), successfully used in different remote control systems, are impossible to be used under the conditions of increased radiation inside the accelerator.

At the present time, the indirect methods of control of magnetic field parameters inside the accelerator are generally used (measurement of dissipated magnetic field intensity, also by vibration magnetometer). They do not meet modern accuracy and reliability requirements. So, the development of equipment for reliable and accurate control of magnetic field parameters, especially inside the accelerator magnetic system circle, is of particular interest.

In the presented work, one of the alternate solutions for the problem of magnetic field parameters control inside the magnetic system circle as well as in close vicinity to the accelerator (below and behind the protective structures) is introduced.

\section{Formulation of the Problem}

For the action electron construction accelerator which consists of 8 magnetic quadrants and 4 homogeneous sectoral electric magnets of the injection tract has been 
tasked — work out the meter for magnetic field parameters control. For this accelerator design of the magnetic field control should be implemented within the magnetic quadrants ring (the center of each quadrant), at the junction between the quadrants (in sections), in the electromagnets, as well as outside of the quadrants to the walls of the bunker and behind the bunker till the protective screen (a minimum of 24 points). The induction of the magnetic field inside the quadrants may be greater than $2.5 \mathrm{~T}$, heterogeneity - the order of $0.25 \mathrm{~T} / \mathrm{cm}$. The inspection time of the parameters magnetic field at each point must be at least one time in $4 \mathrm{~min}$. The measurement accuracy — not more than $1 \%$.

Using the meter with the work principle is based on the phenomenon of nuclear magnetic resonance in the flowing liquid allows to solve the problem. This meter possessed the high measurement accuracy of the magnetic induction. ${ }^{4-6,8,9}$ The value of the induction $B$ in this device is determined by the gyromagnetic ratio of the proton $\gamma_{p}$ and frequency $f_{n}$ of the nuclear resonance. ${ }^{4,8-12}$ The contact between the induction and to previously submitted values is determined by the relation ${ }^{8-12}$

$$
B=C_{p} \times f_{n}
$$

where $C_{p}=2 \pi / \gamma_{p}$ - constant reference meter state special standard for magnetic induction range $2-10 \mathrm{~T}$. For determining of the magnetic field induction $B$ used in the $C_{p}=(2.348740 \pm 0.000007) \times 10^{-8} \mathrm{~T} / \mathrm{Hz}$. This value provides the necessary measurement accuracy. The frequency fn is measured by contemporary devices with high accuracy. Besides that the nuclear-magnetic gauges on the flowing liquid are easy to use. ${ }^{2,4-6,8-12}$

\section{Design of the Magnetometer and Methods of Measurements}

Figure 1 is a block diagram. We developed a two-channel nutation nuclear-resonance magnetometer current fluid. The working fluid is a mixture distillate water-isolated with methanol. The manufacturing liquid fluid has a longitudinal relaxation time $T_{1}=5.93 \mathrm{~s}$. The use of such a medium allows us to place the magnetic field registering sensor (coil nutation) at a distance up to $50 \mathrm{~m}$ from the polarizer and the device registration of the NMR signal.

The flowing liquid from the pump 1 enters the vessel-polarizer 3 on the pipeline. The vessel-polarizer is located in the magnetic system 2 creates a strong magnetic field with induction $B_{p}=1,018 \mathrm{~T}$ in the gap between the pole pieces $d_{n}=15 \mathrm{~mm}$. In this vessel the liquid is magnetized to the value of $M_{p}=\chi_{0} \times B_{p}\left(\chi_{0}-\right.$ static nuclear magnetic susceptibility). The additional description the liquid enters in the flow switch 4 . In his the depending on the point where it is necessary to measure the magnetic field induction $B_{0}$ is switched of flowing stream to the correct channel. At the same time through an electronic key 14 to the corresponding nutation coil 5 is supplied radiofield $H_{1}$ from the nutation generator 15 . The magnetized fluid is arriving in the nutation coil at the connecting sections pipeline 6 . In has under the action of resonance radiofield $H_{1}$ is a change of orientation magnetization $M_{p}$ of 


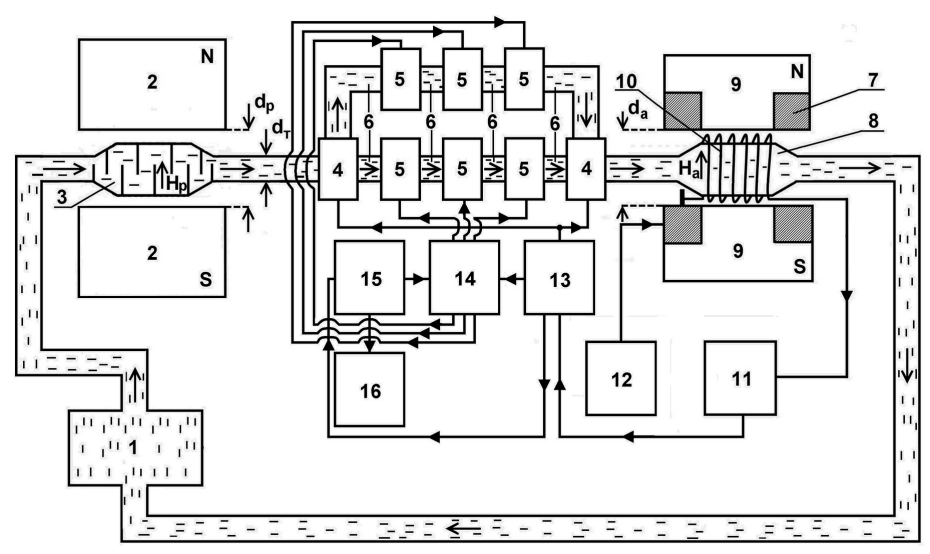

Fig. 1. Block diagram of a nuclear-resonance magnetometer: 1 - pump; 2 - magnet-polarizer; 3 - vessel-polarizer; 4 - switch the flow of the liquid medium; 5 - nutation coil; 6 - connecting portions of the pipeline; 7 - modulation coil; 8 - vessel-analyzer; 9 - magnet-analyzer; 10 NMR signal registration coil; 11 - recording device of the NMR signal; 12 - RF generator; 13 designed to provide processing and management; 14 - electronic keys; 15 - nutation generator; 16 - frequency.

the nuclear moments on the direction of a constant polarizer magnetic field $B_{p}$ inversion of magnetization. ${ }^{4-6,8-12}$ This change of orientation $M_{p}$ after of liquid passage the connecting sections pipeline 6 and the flow switch 4 is fixed of NMR signal registration coil 10 . The coil 10 is a part the newly developed generator circuit vibrations weak (autodyne) which located in the registration device 11. ${ }^{6,12}$

The registration coil is located in the field of the magnet-analyzer 9 with the magnetic field induction $B_{a}=0.182 \mathrm{~T}$, the gap between the poles $d_{a}=10 \mathrm{~mm}$ and the heterogeneity $0.8 \times 10^{-3} \mathrm{~cm}^{-1}$. The full inversion of magnetization $M_{p}$ the vector rotation on $180^{\circ}$ is occurring at the resonant frequency $f_{n}$ radiofield $H_{1}$. This means that at a certain amplitude radiofield $H_{1}$ in the nutation coil in the registration scheme of the NMR signal is observed the maximum inverting the NMR signal. The frequency $\left(f_{H}\right)$ this field $H_{1}$ is equal to the frequency $f_{0}$ of nuclei precession in the field $B_{0}$. Frequency fn associated with the magnetic field $B_{0}$ which is a nutation coil of the relation (1).

The scheme of automatic frequency control (AFC) is adjusting the frequency of nutation generator 15 at the maximum amplitude of the detectable inverted NMR signal. This scheme is located in the device control and data processing 13 .

Based on the decision of the Bloch equations in a rotating coordinate system was obtained the condition across the field $H_{1}$ at which was registered a maximum of inverted NMR signal ${ }^{2,4-6,8-12}$

$$
\gamma H_{1} \frac{V_{n}}{q}=\pi
$$

where $V_{n}$ — the volume of nutation coil 5 (Fig. 1), $q$ — liquid medium expenditure. 
For description of nutation process introduced the magnitude $\Delta t=V_{H} / q$ - the time actions of radiofield $H_{1}$ on the liquid segment of nutation coil. The scheme of phase-locked loop (PLL) is adjusting the amplitude of the RF oscillation generator nutation 15 at the maximum amplitude of the detectable inverted NMR signal. This scheme is located in the device control and data processing 13. The authors have ascertained that for the obtaining of complete magnetization inversion in the nutation coil must be met the relationship (3)

$$
\Delta t>T_{n}
$$

where $T_{n}=1 / f_{n}$ - the period actions of radiofield $H_{1}$.

The obtained result is allowed minimize the length $l_{n}$ of nutation coil up to $4 \mathrm{~mm}$. This is reducing the nutation line width $\Delta f_{n}$. The decrease $\Delta f_{n}$ is increasing of measurement accuracy the magnetic field induction $B_{0}$ and the gradient in the placement zone of nutation coil. For increase of the signal-to-noise ratio of detectable NMR is made the vessel-analyzer 10 of special form. ${ }^{6,12}$ This is allowed to increase the measurement accuracy of the magnetic field.

On the Fig. 2 is presented the outward appearance of manufactured nutation coil 5 with connecting pipeline portions 6 . The coil is wound on plastic frame with outer diameter $2.8 \mathrm{~mm}$, the number of coils 26 (SEW -2 , diameter $0.17 \mathrm{~mm}$ ). The volume of nutation coil is constituting $V_{n}=2.18 \mu \mathrm{l}$, the resistance $R_{n}=0.89 \Omega$ and inductance $L_{n}=43 \mu \mathrm{H}$. For getting a maximum signal-to-noise ratio of detectable NMR signal on the liquid medium expenditure $q=10.0 \pm 0.3 \mathrm{ml} / \mathrm{s}$ was made vesselpolarizer with volume $V_{p}=198 \mathrm{ml}$. In the manufacture of vessel-polarizer were taken into consideration: all volumes of the connecting pipeline portions including the switching elements and etc. ${ }^{2,4-6,10-11}$ This allowed the authors received the NMR signal with signal-to-noise ratio 4.5 in the registration scheme at a given expenditure $q$.

In each of the two channels (between switches stream 4) magnetometer was taken a 12 nutation coils. Eight of them were within the accelerator (center quadrants), eight - in the gaps between quadrants, two — between the quadrant and the casing

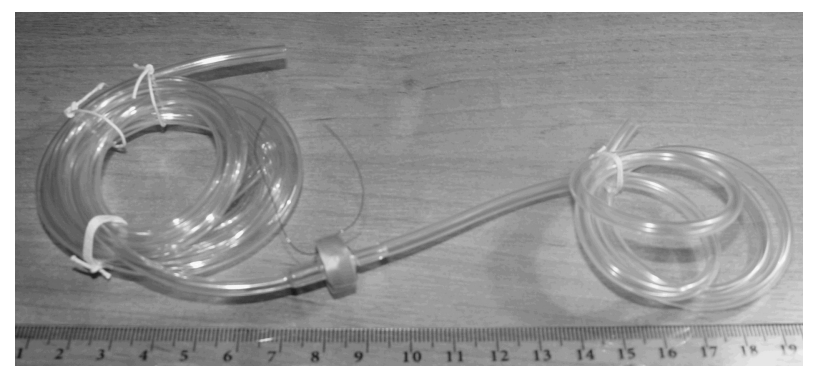

Fig. 2. The outward appearance of nutation coil with connecting pipeline portions. 
bunker, another one - behind housing of bunker, before a protective screens and the last coil was placed behind a behind protective screens. If necessary then the number of coils can be increased, since they do not introduce additional flow resistance in a path of flowing fluid. The periodic poll of nutation coils (supply radio frequency field $H_{1}$ at has with subsequent inclusion $\mathrm{AFC}$ fn on resonance, if appropriate was included phase-locked field $H_{1}$ ) was conducting on specified program. At the resonance frequency $f_{n}$ is determined the magnetic field induction $B_{0}(1)$, and on width of the line nutation $\Delta \mathrm{f}_{n}-\nabla B_{0}(4)$

$$
\nabla B_{0}=\frac{\Delta f_{n}}{\gamma l_{n}} .
$$

The measurement time of induction and the gradient of the magnetic field for specified channel pipeline point between the polarizer 3 and 8 analyzer congestion is not more than 10 seconds.

\section{Measurement Results}

On the Fig. 3(left) is presented as an example of NMR signal is detected after passing through one of the channels without activating it nutation coils. On the Fig. 3(right) is presented the NMR signal at fine-tuning on resonance in the nutation coil, which inverted on 180 degrees in a magnetic field quadrant with induction $\mathrm{B}_{0}=2.2781 \pm 0.0114 \mathrm{~T}$ (nutation frequency $\mathrm{f}_{n}=95681.28 \pm 478.41 \mathrm{~Hz}$ ) by field $\mathrm{H}_{1}$. The nutation line width $\Delta \mathrm{f}_{H}=4298.17 \pm 21.49 \mathrm{~Hz}, \nabla \mathrm{B}_{0}$ is magnitude amounted magnitude $0.2558 \pm 0.0012 \mathrm{~T} / \mathrm{cm}$ in the placement zone of nutation coil.

The measuring cycle of frequency $\mathrm{f}_{n}$ with the setting on the response repeated 20 once for each point of placing the nutation coil. After this determined the mean value, standard deviation and error of measurement accuracy with credibility $95 \%$.
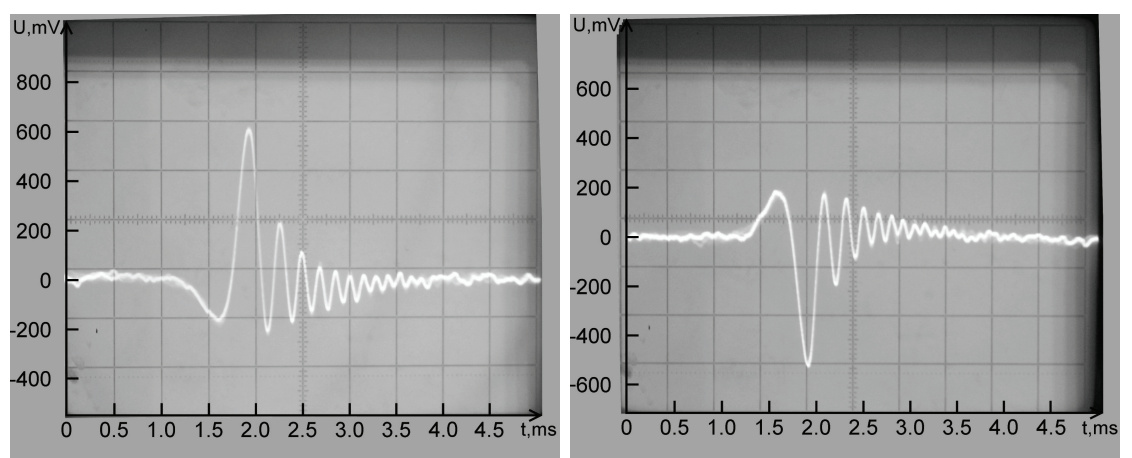

Fig. 3. (left) — The shape of the NMR signal line on output of the registration scheme 11 for fabricated made of the liquid medium at a temperature $\mathrm{T}=288.4 \mathrm{~K}, \mathrm{f}_{\bmod }=50 \mathrm{~Hz}$. (right) - The shape of the NMR inverted signal line on output of the registration scheme 11 for fabricated made of the liquid medium at temperature $\mathrm{T}=288.4 \mathrm{~K}, \mathrm{f}_{m o d}=50 \mathrm{~Hz}, \mathrm{f}_{n}=95681.28 \mathrm{~Hz}$. 
Table 1. The induction and gradient of magnetic field in different points of accelerator and also beyond the bunker accelerator.

\begin{tabular}{ccc}
\hline \multicolumn{3}{c}{ Nutation NMR Magnetometer } \\
\hline Location point & $\mathrm{B}_{0}, \mathrm{~T}$ & $\nabla \mathrm{B}_{0}, \mathrm{~T} / \mathrm{cm}$ \\
\hline 1 & $2.2781 \pm 0.0114$ & $0.2558 \pm 0.0012$ \\
2 & $0.4718 \pm 0.0023$ & $0.1185 \pm 0.0006$ \\
3 & $(0.1094 \pm 0.0004) \times 10^{-1}$ & $(0.1392 \pm 0.0005) \times 10^{-2}$ \\
4 & $(0.2543 \pm 0.0009) \times 10^{-2}$ & $(0.2234 \pm 0.0008) \times 10^{-3}$ \\
5 & $(0.6259 \pm 0.0025) \times 10^{-4}$ & $(0.8525 \pm 0.0034) \times 10^{-5}$ \\
\hline & Gaussmeter $1-\mathrm{ST}$ & \\
\hline Location point & $\mathrm{B}_{0}, \mathrm{~T}$ & $\nabla \mathrm{B}_{0}, \mathrm{~T} / \mathrm{cm}$ \\
\hline 3 & $(0.1086 \pm 0.0011) \times 10^{-1}$ & $(0.1371 \pm 0.0022) \times 10^{-2}$ \\
4 & $(0.2534 \pm 0.0026) \times 10^{-2}$ & $(0.2211 \pm 0.0034) \times 10^{-3}$ \\
5 & $(0.6251 \pm 0.0062) \times 10^{-4}$ & $(0.8493 \pm 0.0138) \times 10^{-5}$ \\
\hline & Gaussmeter DX-160 & \\
\hline Location point & $\mathrm{B}_{0}, \mathrm{~T}$ & $\nabla \mathrm{B}_{0}, \mathrm{~T} / \mathrm{cm}$ \\
\hline 3 & $(0.1095 \pm 0.0004) \times 10^{-1}$ & $(0.1393 \pm 0.0011) \times 10^{-2}$ \\
4 & $(0.2546 \pm 0.0010) \times 10^{-2}$ & $(0.2239 \pm 0.0018) \times 10^{-3}$ \\
5 & $(0.6261 \pm 0.0025) \times 10^{-4}$ & $(0.8532 \pm 0.0069) \times 10^{-5}$ \\
\hline
\end{tabular}

For all investigations at varying heterogeneity degrees of magnetic field the measurement error does not exceed $0.5 \%$. The error of definition the nutation line width $\Delta \mathrm{f}_{n}$ is defined similarly.

The measured values of induction and gradient of magnetic field in various points of the accelerator and behind outside the bunker accelerator are showed in Table 1, where the locations of the measurement points are: 1 - in the center of the quadrant, 2 - in the gap between quadrants, 3 - under the hopper hatch accelerator, 4 - between the hopper and the accelerator shields, 5 - in the area of deployment of personnel, instrumentation and remote control accelerator. For comparison in Table 1 shows the measuring the magnetic field inhomogeneity and made industrial gauges (gaussmeter 1 - ST and DX - 160) in the available personnel points of the accelerator.

From-due to limited of technical capabilities in developed magnetometer by us were used only two channels of flow switches 4 . The performed measurements have shown that the best option - it is a separate channel allocation for each quadrant of the magnetic system. This is reducing the measurement time and improved the signal-to-noise ratio of the recorded NMR signal. But significantly gets rough the design of the channel selector. Currently, import switches are available for 10 channels. It is not enough because there are accelerators in which are used 32 quadrants. Therefore in the current situation having applied authors the use of one channel for measuring current flow in several quadrants is fully justified. The design of the 
device can be simplified by eliminating the magnet polarizer. The feature of polarizer can perform the strong accelerator magnetic fields.

\section{Conclusion}

The carried out experimental researches are showing that the developed prototype of multichannel magnetometer allows to make measurements of strong magnetic field parameters with error not more than $0.5 \%$ at a sensitivity $10^{-10} \mathrm{~T} / \mathrm{Hz}^{1 / 2}$ in automatic regime. The received results of the measurements are allowing to correct a quadrants position of magnetic system accelerator and effectively measure the magnetic environment in indoors near with accelerator.

The developed device can also be used for measurements of magnetic field with induction $\mathrm{B}_{0}$ above $0.7 \mathrm{~T}$ and the heterogeneity of $0.06 \mathrm{~T} / \mathrm{cm}$ near with marine power plants on military ships. The modern quantum magnetometers can not work in such fields - problems with igniting lamp, etc. The other model magnetometers are not ensuring the necessary measurement accuracy in conditions of strong electromagnetic interference.

For parameters control of magnetic field in other models of accelerator is necessary is increasing the distance up to $100 \mathrm{~m}$ from the measurement point to the place of registration NMR signal. For it is planned to increase the field induction $\mathrm{B}_{p}$ of magnet-polarizer before $1.5 \mathrm{~T}$ through the use of more powerful magnetic materials. And also will have manufacturing the liquid medium with $\mathrm{T}_{1}>7 \mathrm{~s}$ and a viscosity of $\eta<2.5 \times 10^{-3} \mathrm{~Pa} \cdot \mathrm{s}$ in the temperature range from 278 to $313 \mathrm{~K}$.

\section{References}

1. E. B. Aleksandrov and A. K. Vershovskii, Uspekhi Fizicheskih Nauk 52, 573 (2009).

2. R. Fukuda and A. Hirai, J. Phys. Soc. Japan 47, 1999 (1979).

3. M. D. Prouty, R. Johnson, N. Hrvoic and A. K. Vershovskiy, Geophysical applications, in Optical Magnetometry, ed. O. Budker and D. F. Kimball (Cambridge University Press, Cambridge, 2013), p. 319.

4. V. V. Davydov and V. V. Semenov, Instruments and Experimental Techniques 41, 151 (1999).

5. V. V. Davydov and V. V. Semenov, Journal of Communications Technology and Electronics 44, 1528 (1999).

6. V. V. Davydov, V. I. Dudkin and A. Yu. Karseev, Tech. Phys. Journal 85, 138 (2015).

7. V. I. Dudkin et al., Journal of Communications Technology and Electronics 53, 368 (2008).

8. V. V. Davydov, V. I. Dudkin and A. Yu. Karseev, Tech. Phys. Lett. 40, 895 (2014).

9. V. I. Dudkin and L. N. Pakhomov, Kvantovaya Elektronika (Polytechnical Institute Publ., Saint-Petersburg, 2012).

10. V. V. Davydov, E. N. Velichko, V. I. Dudkin and A. Yu. Karseev, Measurement Techniques 57, 684 (2014).

11. R. E. Habbach et al., Rev. Sci. Instr. 80, 428 (2009).

12. V. V. Davydov, V. I. Dudkin and A. Yu. Karseev, Optical Memory \& Neural Networks 22, 112 (2013). 